\title{
GRAVITATIONAL ACCELERATION AND FORBIDDEN LINE WIDTHS IN SEYFERT GALAXIES
}

\author{
MARK WHITTLE \\ Astronomy Department \\ University of Virginia \\ Charlottesville VA 22903 \\ U.S.A.
}

There now exists extensive data on the forbidden line widths in Seyfert galaxies. While a few Seyferts have anomalously broad lines with FWHM $\sim 500-1000 \mathrm{~km} \mathrm{~s}^{-1}$ (e.g. NGC 1068, MKN 3, MKN 78), the majority have narrower lines with FWHM $\sim 200-400 \mathrm{~km} \mathrm{~s}^{-1}$. Although these line widths are well known to be of Doppler origin, the physical processes responsible for accelerating the gas have yet to be clearly identified. Processes associated with the activity itself are suggested by the large linewidths of the broadest profiles as well as by the presence of a correlation between [OIII] FWHM and steep spectrum radio luminosity. On the other hand, the prevelence of narrower lines and total absence of correlation between [OIII] FWHM and overall level of activity (Seyfert 2s, Seyfert 1s, and QSOs) suggests that more normal, possibly gravitational, processes are important. To test this latter possibility, it is necessary to look for explicit correlations between [OIII] FWHM and diagnostics of galactic gravitational potential.

Figure 1 shows [OIII] FWHM plotted against observed galactic rotation amplitude (defined from long slit optical observations or single beam HI profiles) for about 60 Seyferts. While objects with linear radio morphology and relatively high power (crosses) tend to have anomalously broad lines, the correlation for the remaining $75 \%$ of the sample is extremely significant $(\mathrm{R}=0.6, \mathrm{P}$ (null) $>99.999 \%$, two tailed) and has a best fit line lying along $\mathrm{y}=$ $\mathrm{x}$. Thus, not only does [OIII] FWHM correlate with the observed rotation amplitude, it is actually equal to it. The simple conclusion is that for most Seyferts the primary acceleration mechanism is gravitational. For Seyferts with high power linear radio sources, there is an additional acceleration process which very probably results from the interaction of a jet with the interstellar medium. A more detailed description of this latter phenomenon can be found in Whittle et al. (1987).

How can the rotation amplitude, which is defined at large radii, be linked to highly nuclear velocities measured within 2" - 3" apertures ? There are two general possibilities. First, the rotation curve may remain high right in to the nucleus so that rotational smearing gives apparently broad lines. Second, even if rotation is low in the nucleus, the bulge dispersion velocities are still high, and these are in turn related to the disk rotation amplitude (Whitmore and Kirshner 1981). Plotting [OIII] FWHM against stellar dispersion inferred from the rotation amplitude shows a good correlation and confirms that the [OIII] FWHM are similar to the stellar dispersion velocities (FWHM). An independent demonstration of this is shown in Figure 2 in which [OIII] FWHM is plotted against bulge absolute B magnitude, derived from total magnitudes and bulge/total ratios defined for normal galaxies of the same Hubble type. There is a moderately strong correlation ( $\mathrm{P}($ null $) \sim 99.99 \%)$ with best fit line close to the classic $\mathrm{L} \sim \sigma^{4}$ relation for stars (shown as the dashed line). 
[OII] FWHN VS OBSERVED GALAXY ROTATION

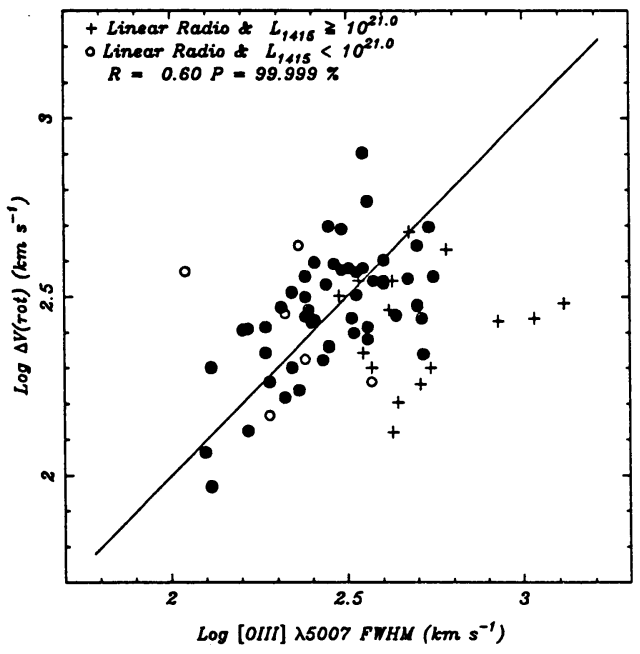

Figure 1

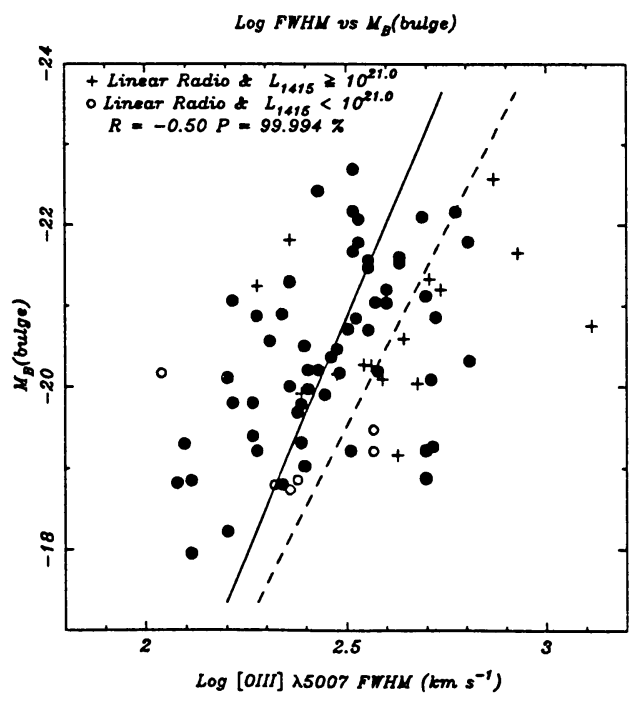

Figure 2

In principle it is possible to distinguish between smearing of disk rotation and more isotropic dispersion velocities. For rotation the strongest correlation should be with observed rotation amplitude while for dispersion it should be with inclination corrected or "true" rotation amplitude. In fact, for Seyferts with reliably measured inclinations, there is no difference in correlation strength between these two rotation measures, suggesting that the NLR velocities combine both a disk component and a more isotropic component.

Finally, it seems that similar correlations are present but weaker for [OIII] base width and wing width parameters (FW20 and IPV10). Unfortunately, interpretation of this is not straightforward. Although non-gravitational velocities may dominate on the small (< $10 \mathrm{pc}$ ) scales sampled by the [OIII] base and wings, it is also possible that gravitational velocities are important but that they are only weakly related to the values measured on the larger (arcsec) scales. It is also possible that the wing parameters, while well defined observationally, may be poorly defined physically, since they depend sensitively on the relative strength of the line core and base.

To summarise, the correlations with rotation amplitude and bulge magnitude strongly suggest that the [OIII] FWHM in most Seyfert galaxies are determined by motion in the gravitational field of the bulge. For Seyferts with powerful linear radio sources, additional acceleration results from a jet interaction with the interstellar medium. Consideration of the inclination dependence of these correlations implies that the velocity field is neither pure rotation nor pure dispersion. Similar correlations for the base and wing velocities are somewhat weaker, although it is not clear what this signifies.

Whitmore, B.C., and Kirshner, R.P., 1981, Astrophys. J., 250, 43.

Whittle, M., Pedlar, A., Meurs, E.J.A., Unger, S.W., Axon, D.J., and Ward, M.J., 1987

Astrophys J., 326, 125. 


\section{DISCUSSION}

\section{2 galaxies? \\ KHACHIKIAN Did you find any difference between the Seyfert 1 and Seyfert}

WHITTLE: No. The early studies which found systematic differences between [O III] FWHMs for Seyfert 2 and Seyfert 1 galaxies were influenced by the selection process of the Markarian Seyferts, which favor rather luminous Seyfert 2s. The more comprehensive lists of [O III] FWHM now available show no systematic differences (Whittle, MNRAS, 213, 33, 1985).

DJORGOVSKI Your correlation between the nuclear ([O III] width) and largeradius (rotation-curve amplitude) velocity measures seems to imply that all spiral galaxies have very similar dynamical structures over a large range in radius. This is a highly nontrivial statement, and perhaps it will be an important tool to study not only the active nuclei, but also some hard-to-probe global properties of galaxies. Perhaps this dynamical similarity derives from the power-law shape of galactic halos. I would guess that you can lower the scatter of this relation by using the inner slope of the rotation curve as the second parameter.

WHITTLE I agree that the similarity of near nuclear and far disk velocities is remarkable. It has been known to be approximately true using normal gas or star velocity tracers and has come to be called the "bulge-disk-halo conspiracy." I am not sure whether the [O III] FWHM relations will be any better at probing the conspiracy than the normal gas or star observations - though possibly easier to measure observationally.

G. BURBIDGE How do you know that the rotation measures apply to the galaxy? Did you see extended emission in the Seyfert spectra?

WHITTLE Yes, the rotation amplitudes were only used if there was evidence of turnover and an approximately flat rotation curve. In any case, most of the rotation amplitudes were taken from large-beam $\mathrm{H}$ I measurements in which the disk rotation dominates the profile. 